\title{
Révision de la Loi du 20 décembre 1988 : plate-forme commune de propositions émanant des sociétés savantes, organismes et associations de malades
}

La loi Huriet-Sérusclat a été votée en décembre 1988. Elle a autorisé la recherche clinique en France, jusque-là clandestine et à certains égards illégale. Aucun médecin ne peut remettre en cause le bien-fondé d'une législation qui organise la recherche clinique et garantit la sécurité des individus qui s'y prêtent. Mais la montée en charge de la recherche clinique dans notre pays a mis en évidence des difficultés et des blocages inhérents à la loi et à ses décrets d'application. La raison majeure en est que la loi était initialement destinée à l'évaluation des nouveaux médicaments, et qu'elle avait décalqué les lourdes procédures des «bonnes pratiques cliniques» développées par lindustrie pharmaceutique. Ce n'est que secondairement, lors de la présentation de son projet par Claude Huriet devant la commission des affaires sociales du Sénat, que le champ d'application de la loi a été étendu à toute la recherche biomédicale, médicamenteuse ou non, avec ou sans bénéfice individuel direct, sur malade ou sujet sain, et cela sans adaptation de son architecture à ces nouveaux domaines. La conséquence, bien apparente aujourd'hui, est que l'évaluation des médicaments, pour lesquels la loi a été faite, s'effectue convenablement, à la satisfaction de l'industrie pharmaceutique. Mais dans tous les autres domaines qui entrent aussi dans son champ d'application, telles la recherche non médicamenteuse sur volontaire sain (physiologie), la recherche sans bénéfice direct sur patients (physiopathologie), la comparaison de pratiques, les études médico-économiques, la recherche sur patient dit "incompétent", les études génétiques, etc., la loi est si peu adaptée que la recherche s'y effectue encore clandestinement ou s'est interrompue totalement. Cette situation est d'autant plus inacceptable et non éthique qu'elle concerne des affections graves, souvent mortelles, et pour lesquelles des progrès sont indispensables.

Cette "plate-forme» a été signée par la quasi-totalité des sociétés savantes françaises, la Conférence des Doyens, les deux conférences de Présidents de CME, témoignant bien de l'extrême préoccupation de la communauté médicale nationale sur ce sujet. Plusieurs associations de malades l'ont également signée, montrant ainsi que ceux-ci ont bien perçu et partagent l'objectif de progrès et d'amélioration de la santé publique en France et non les intérêts des seuls médecins universitaires. Le but est bien d'améliorer la sécurité des individus qui se prêtent aux essais, car il est pour le moins douteux qu'une procédure inadaptée soit une garantie de sécurité pour eux. La révision des lois bioéthiques et l'introduction dans le droit national de la récente directive européenne sur ce sujet, signée à Strasbourg en décembre dernier, vont offrir une occasion unique de faire évoluer la loi de décembre 1988. La communauté médicale attend que soit enfin entreprise cette indispensable adaptation de la loi à la totalité de son champ d'application. es sociétés savantes, associations et organismes signataires de cette plate-forme: - rappellent que l'amélioration de la santé publique passe par la progression des connaissances et que celle-ci, obtenue grâce à la recherche clinique, est une des missions prioritaires de la médecine d'aujourd'hui ;

- soulignent que la loi du 20 décembre 1988 modifiée, dite loi Huriet-Serusclat, a eu un indiscutable effet bénéfique sur la protection des personnes qu'elle a garantie, et sur la recherche clinique française qu'elle a organisée;

- constatent aussi que la loi et la réglementation qui en a découlé, conçues initialement pour permettre l'évaluation et l'enregistrement en 
France des médicaments innovants, ne sont pas adaptées à de nombreuses situations qui entrent pourtant dans son champ d'application, considérablement élargi lors du débat parlementaire de 1988.

Soulignons :

- des procédures trop lourdes pour la simple comparaison de stratégies diagnostiques, de traitements ou de dispositifs déjà validés et utilisés en routine;

- l'impossibilité de réaliser des études physiopathologiques (sans bénéfice individuel direct: sans BID) dans des situations qui ne permettent pas de recueillir le consentement des patients, la gravité (choc, coma, atteintes neurologiques centrales, etc.) exige pourtant que des recherches leur soient consacrées ;

- des lourdes contraintes propres aux études sans BID, à l'origine prévues pour les essais thérapeutiques de phase I ou II sur volontaires sains, se révèlent inadaptées à de nombreuses autres situations, telles les recherches sur échantillon sanguin lors des soins courants;

- observent que dans ces cas une procédure lourde et inadaptée nuit en fait à la protection des patients, certaines investigations pourtant utiles ne se faisant pas, et d'autres s'effectuant probablement encore dans la clandestinité;

- souhaitent qu'à l'occasion de la révision des lois bioéthiques prévue en 2001 soient pris en compte les demandes réitérées de clarification et/ou de simplification émanant de sociétés savantes françaises [1, 2], ainsi que l'avis $n^{\circ} 58$ du CCNE (octobre 1998), qui avait recensé certaines de ces difficultés et proposé des modifications législatives ou réglementaires, reprenant d'ailleurs les recommandations déjà émises en 1992 dans un rapport de l'IGAS.

\section{Ces sociétés savantes, associations et organismes proposent}

\author{
Une procédure allégée \\ pour la comparaison \\ de pratiques validées
}

1. Une procédure allégée devrait pouvoir 1032 rer les tests diagnostiques, traitements ou dispositifs déjà autorisés et utilisés en routine, et pour lesquels aucune contrainte particulière ne serait requise s'ils étaient mis en œuvre isolément, en dehors de l'essai comparatif proposé. Considérant que ce type de recherche s'applique à des dispositifs, traitements et tests diagnostiques déjà validés et de toute façon nécessaires, et sous réserve qu'il n'y ait aucun risque ou inconvénient supplémentaires liés à l'étude, on pourrait envisager ici d'utiliser plus systématiquement la possibilité réglementaire (circulaire DPHM 01/08 n 90-4 du 24 octobre 1990) de fusionner les rôles de l'investigateur et du promoteur, et d'inscrire le régime de responsabilité dans le droit commun. L'allégement de la procédure qui est souhaité ne dispense en aucun cas de l'information des patients, ni du recueil de leur consentement. Les projets resteraient soumis à l'approbation des CCPPRB, dont le rôle majeur serait ici d'évaluer loyalement, en toute indépendance, la balance risques/bénéfices. Pour renforcer encore le rôle des CCPPRB dans cette procédure, le suivi de ces projets pourrait leur être confié.

2. La suppression de l'obligation pour le promoteur, contenue dans l'article R-2038, de fourniture gratuite des médicaments et de financement des frais (s'agit-il ici véritablement d'un surcoût de la recherche, et non pas plutôt du simple coût de soins par ailleurs nécessaires?). Cette obligation devrait pouvoir être levée, dès lors que la recherche concerne un traitement ou une procédure déjà autorisés, dans les indications de leur AMM et dont le patient a de toute façon besoin. Dans ces situations, le promoteur est en fait habituellement institutionnel: hôpital le plus souvent, société savante ou association parfois.

\section{La reconnaissance du patient «incompétent»}

3. La loi devrait prendre en compte les situations d'incompétence des patients dont l'altération des fonctions cognitives ne leur permet pas de consentir. Ces situations, différentes de l'urgence proprement dite (article L. 209-9) sont rencontrées certes fréquemment en réanimation, mais aussi en gériatrie, en pédiatrie, en psychiatrie, en neurologie. Une «personne de confiance ", désignée à l'avance par le patient lui-même, ou, à défaut, un membre de sa famille, devraient pouvoir se substituer au patient, par extension de la disposition déjà prévue à l'article L. 209-9 en cas d'urgence.

\section{Une meilleure appréciation} des études sans bénéfice individuel direct

La loi de décembre 1988 oppose les essais avec et sans BID. En pratique, comme l'a souligné récemment le CCNE dans son avis $\mathrm{n}^{\circ} 28$, cette distinction est souvent difficile à établir avec clarté, elle est exposée à des manipulations et est remise en question aujourd'hui au plan international [3]. La dernière révision de la déclaration d'Helsinki (Association médicale mondiale) ne la retient plus [4]. Surtout, la prise en compte littérale de cette distinction peut en fait aboutir à une diminution de la protection des patients: si la notion de BID est par trop mise en avant, elle peut conduire à faire courir aux patients des risques excessifs; inversement, s'il n'y a pas de BID, certaines études pourtant indispensables (physiopathologiques notamment) demeureront interdites dans de nombreuses circonstances, notamment en urgence. 4. Des études à visée cognitive, sans BID, doivent pouvoir être réalisées chez des patients incapables de consentir. Il est, en effet, certainement non éthique, voire absurde, de priver du bénéfice de la recherche biomédicale les populations de patients les plus vulnérables et qui, précisément, en auraient le plus besoin. L'information serait alors délivrée, et le consentement recherché, auprès d'une "personne de confiance» (représentant, mandataire), désignée à l'avance par le patient, ou, à défaut, auprès de membre(s) de sa famille. Le renforcement de la protection de ces patients vulnérables pourrait être envisagé, par exemple par l'obligation d'obtention d'une autorisation préalable auprès de l'autorité admi- 
nistrative compétente. Le recours au juge judiciaire pour la désignation du représentant du patient, proposé dans ces circonstances par certains juristes, est ressenti par les acteurs de terrain comme impraticable et totalement irréaliste.

5 . Une procédure particulière doit être définie pour la recherche cognitive sans BID, mais n'imposant aucun risque particulier, telle par exemple, l'utilisation pour la recherche de «fonds de tube», qui ne nécessitent pas de prélèvement supplémentaire lors de soins courants. Une attention particulière doit être accordée aux études génétiques qui, de fait, reposent sur l'analyse d'échantillons sanguins. En effet, l'évolution rapide des connaissances dans ce domaine et l'impact croissant de cette discipline en médecine justifient de pouvoir collecter, dans des situations cliniques très diverses, de simples prélèvements sanguins; par ailleurs, la possibilité de recueillir des échantillons sanguins de membre(s) de la famille du patient concerné par une maladie à composante génétique devrait également être examinée. Ces prélèvements peuvent servir à constituer des banques de cellules ou d'ADN. Dans ces cas, l'anonymisation des échantillons et le consentement des patients pour une utilisation ultérieure doivent être prévus par le protocole.

\section{L'organisation de l'information a posteriori des patients ou de leurs ayants droit}

6. L'information des patients ou de leurs ayants droit devrait être organi- sée à l'issue de l'essai. En cas de complications, une procédure permettant de relever le médecin-investigateur du secret médical, si besoin est, devrait être mise au point.

Le régime de responsabilité des essais avec BID (aujourd'hui celui de la faute présumée, article L. 209-7) devrait être aligné sur celui des essais sans BID: indemnisation des conséquences dommageables imputables à la recherche, même sans faute.

\section{Renforcement du rôle des CGPPRB}

7. Les sociétés savantes, associations et organismes signataires considèrent que les CCPPRB, plutôt que d'avoir à tracer la frontière entre les essais avec et sans BID, bien souvent imprécise et arbitraire, devraient plutôt mettre l'accent sur l'esprit de la loi, qui est de garantir et de renforcer la protection des patients. Leur mission est bien d'évaluer de façon loyale et scientifique la balance bénéfices/ risques de chacun des projets qui leur sont soumis, et plus spécifiquement de chaque élément constitutif de ces projets, qu'ils soient avec ou sans BID. Le suivi des projets que les CCPPRB ont approuvé devrait être organisé. En contrepartie de ce rôle accru des CCPPRB, leur évaluation régulière par la tutelle pourrait être instituée.

\section{Conclusions}

En règle générale, et en facteur commun à toutes ces propositions, les sociétés savantes, associations et organismes signataires souhaitent, dans certains cas précis, l'allégement d'une procédure lourde et complexe, certes légitime en cas d'essai portant sur des thérapeutiques innovantes ou des procédures diagnostiques invasives, mais inadaptée et dissuasive dans les situations décrites plus haut. Leur but n'est pas de se soustraire à l'obligation d'information et de consentement des patients sollicités, ni d'échapper au contrôle d'une instance indépendante, le CCPPRB, dont ils souhaitent, au contraire, renforcer le rôle et les responsabilités. Enfin, il va de soi que les recherches décrites ici restent menées dans l'esprit des bonnes pratiques cliniques

\section{RÉFÉRENCES}

1. Cales P, Henrion J, Pascal JP, Valla D, Lapuelle J, Lunel F, Poynard T. Proposition de charte de déontologie en recherche clinique. Gastroenterol Clin Biol 1998; 22: 121-6.

2. Lemaire F, Langlois A, Outin H, Rameix S. Groupe de travail de la commission d'éthique de la société de réanimation de langue française. Recherche clinique en réanimation: problèmes liés à l'application de la loi du 20 décembre 1988. Reanim Urg $2000 ; 9$ : 215-23.

3. CCNE. Rapport et recommandations $n^{\circ} 58,12$ juin 1998. Consentement éclairé et information des patients qui se prêtent à des actes de soin ou de recherche. Cahiers du Comité consultatif national d'éthique 1998; $17: 3-22$.

4. Levine RJ. The need to revise the declaration of Helsinki. N Engl J Med 1999; 34: 5314.

5. Déclaration d'Helsinki de l'association médicale mondiale. Octobre 2000. Site internet: http//www.wma.net. 